\title{
La teoría del cine en la región latinoamericana y las políticas de la investigación
}

\section{Film Theories in Latin America and Research Policies}

\author{
Lauro Zavala \\ Universidad Autónoma Metropolitana - Xochimilco (México) \\ zavala38@hotmail.com
}

\section{Resumen}

En este trabajo se exploran las condiciones que determinan la insularidad de los estudios sobre teoría y análisis del cine en la región iberoamericana, que explican la ausencia de teorías del cine de alcance universal producidas en la región. Esta insularidad tiene tres vertientes: (1) la Insularidad Interna existe entre los países de la región, no sólo en términos de la distribución de las películas, sino sobre todo en la distribución de los libros, las revistas especializadas y los mismos investigadores y sus ideas; (2) la Insularidad Externa existe en relación con la tradición anglosajona (en Canadá, Inglaterra y Estados Unidos), lo cual significa un total aislamiento de una fuerte tradición didáctica y de profesionalización de la investigación teórica, y por último (3) la Insularidad Disciplinaria, al no contar en la región con una fuerte tradición en las aproximaciones humanísticas a los estudios sobre cine, a expensas de la abrumadora tradición de estudios en las ciencias sociales (más del 90\% de la producción bibliográfica en los últimos 40 años). Al final del trabajo se proponen algunas estrategias para contrarrestar estas formas de insularidad.

\section{Palabras clave}

Políticas de la Investigación; Estudios Cinematográficos; Teoría del Cine: Epistemología Fílmica; Cine Latinoamericano

\begin{abstract}
In this paper I explore the conditions and consequences of the isolation of film scholarship in Latin American universities. This isolation is threefold: (1) internal isolation between the countries of the region, not only in the distribution of films in any format, but mostly a bibliographic isolation between researchers, books, and scholarly journals between these countries; (2) external isolation between Latin American scholars and colleagues in English-speaking countries (U.S, Canada, and England), and therefore an isolation of a very strong didactic and casuistic tradition in scholarly research, teaching, and formation of researchers; and (3) professional isolation between humanistic research on film and the research in social sciences
\end{abstract}


(where the latter is over $90 \%$ of all film research projects in the region). In the last section of the paper I point out some strategies that might counterbalance this general situation.

\section{Keywords}

Politics of Research; Film Studies; Film Theory; Film Epistemology; Latin American Cinema.

\section{Introducción}

La situación de los estudios sobre cine en América Latina ha estado dominada en los últimos 50 años por tres características que han impedido establecer un diálogo productivo con la tradición internacional en los estudios sobre historia, teoría y análisis, de tal manera que se pueda hablar de una tradición epistemológica de carácter universal producida en lengua española.

Estas características son: una insularidad entre los países de la región (lo cual afecta especialmente los estudios sobre la historia del cine), una insularidad con el exterior (lo cual afecta especialmente a la teoría del cine) y una presencia aplastante de la tradición historiográfica por encima de las aproximaciones humanísticas (lo cual afecta especialmente a la crítica cinematográfica y el análisis de secuencias).

Estas características han marcado la naturaleza epistemológica de los estudios en la región, y son compartidas con gran parte de los estudios humanísticos, especialmente en los campos que le resultan más próximos, como la fotografía, las artes escénicas, las artes visuales, la musicología y (sobre todo) la literatura. Esta condición no sólo afecta al terreno de la epistemología, sino también los terrenos de la lógica argumentativa (tan importante para el análisis de secuencias) y de la articulación entre la ética y la estética del cine.

A continuación se exploran, de manera muy general, las características de cada una de estas tres formas de insularidad en los estudios cinematográficos de la región.

\section{La insularidad interna}

La insularidad interna en los estudios cinematográficos en América Latina consiste en el desconocimiento que existe en cada uno de nuestros países de lo que se produce en el resto de la región en el campo de la investigación universitaria y de la crítica en general. En otras palabras, todos sabemos que los estudios sobre cine 
producidos en cada uno de nuestros países difícilmente circulan entre los demás países de la región, de manera paralela a lo que ocurre con la distribución de los materiales cinematográficos. Así como en México no se conocen las películas producidas en Argentina, Brasil, Colombia, Cuba o Venezuela (con excepciones extremadamente raras), de la misma manera los estudios sobre cine producidos en cada país latinoamericano sólo en casos excepcionales llegan a circular más allá de las fronteras de cada país.

Es necesario que un investigador (o un director de cine) logre publicar sus libros (o producir sus películas) en Europa o en los Estados Unidos para que estos materiales sean distribuidos no sólo en su propio país de origen, sino también en los países de la región iberoamericana. Se podría decir que un fenómeno similar al de los directores Alejandro González Iñárritu, Guillermo del Toro y Alfonso Cuarón o los actores Gael García y Salma Hayek existe en el terreno de la epistemología del cine. Éste es el caso de los investigadores argentinos Mario Onaindia, Sergio Wolf y Eduardo Russo (con sus estudios sobre el guión en el cine clásico, sobre cine y literatura, y el Diccionario del cine, respectivamente, publicados todos ellos en Paidós), el mexicano José Felipe Coria o el brasileño Arlindo Machado (con sus reflexiones sobre el espectador de cine, también en Paidós, y el recuento de las secuencias canónicas en la historia del análisis cinematográfico, este último publicado por Gedisa). ${ }^{1}$

Sólo en casos excepcionales los trabajos de nuestros investigadores llegan a ser conocidos en la misma región. A partir de 2008 merece ser notado el trabajo de la editorial Manantial, en Buenos Aires, pues no sólo publica autores latinoamericanos que producen reflexiones sobre historia, teoría y análisis de cine, sino que ha logrado establecer una red de distribución a la que han estado ajenos los editores más importantes sobre estos terrenos en la región, como Era (para los trabajos sobre historia del cine mexicano de Emilio García Riera), la Universidad de Guadalajara (para los estudios monográficos sobre directores) o la Universidad Nacional (UNAM), para mencionar el caso mexicano.

Además, esta misma editorial argentina ha dado a conocer el trabajo de uno de los teóricos más destacados que ha producido América Latina, que es el brasileño Ismail Xavier. Varias décadas después de haberse publicado en Brasil su trabajo seminal sobre la transparencia en el cine de ficción (en portugués, en 1984), los hablantes de español podemos tener acceso a su trabajo, acompañado además por sus propias reflexiones sobre lo que ha ocurrido durante estos años en relación con este concepto central de la epistemología europea del cine clásico. ¿Cuántas décadas más tendremos que esperar para que los colegas brasileños conozcan los

1 Ver al final las referencias bibliográficas de los libros que se mencionan a lo largo de este trabajo. 
trabajos de los argentinos Russo, Onaindia, Faretta y otros investigadores de la región que escriben en español?

A la insularidad lingüística y editorial de los trabajos de los investigadores de la región se suma la insularidad de los archivos fílmicos. Sólo puede haber investigación ahí donde hay acervos actualizados, eficientes y accesibles. Considerar la importancia estratégica que tendría la difusión inmediata y permanente de los materiales audiovisuales que se producen en cada uno de los países de la región es algo que no ha recibido la suficiente atención en los respectivos ministerios de cultura, en su conjunto, en la región iberoamericana, aunque ya fue insistentemente señalado en el reciente Congreso Iberoamericano de la Cultura, realizado en México en octubre de 2008 y auspiciado por la Cumbre de Presidentes de Iberoamérica. Aunque en ese congreso se concluyó que el problema más grave del cine latinoamericano es su falta de distribución, sin embargo no se llegó a concretar ningún proyecto de trabajo en conjunto por parte de los respectivos ministerios de cultura.

No se puede hablar de una epistemología de los estudios cinematográficos en América Latina mientras no exista un cine latinoamericano. Y nunca ha existido un cine latinoamericano por la sencilla razón de que en ningún país se conocen las películas producidas en los demás países de la región. ${ }^{2}$

Tal vez sorprenda a los colegas brasileños saber que en México no se conoce el trabajo del Cinema Novo. Nadie ha visto las películas de Glauber Rocha. Tal vez sorprenda a los colegas argentinos saber que en México no se conocen películas canónicas como Tiré dié. No se conoce el trabajo de Jorge Sanjinés. Nadie sabe qué es el Grupo Ukamau, de Bolivia, a pesar de que sus libros fueron publicados en México en la década de 1980. Y seguramente en Venezuela o en Cuba no se conocen muchas de las películas canónicas de los mexicanos Roberto Gavaldón o Ismael Rodríguez, que son parte de la cultura audiovisual de todo cinéfilo mexicano.

La posibilidad de establecer comparaciones entre el cine cómico de Oscarito (en Brasil) y Tin Tan (en México) sólo será posible cuando exista una Red Iberoamericana de Videotecas Públicas en el interior de cada uno de los países latinoamericanos. Mientras esto no exista, los cinéfilos en general y los investigadores especializados (que no son sino una especie de cinéfilos altamente calificados) tendrán que conformarse con lo que se llega a colgar en YouTube y en

\footnotetext{
2 Una excepción a esta regla fue la notable distribución y aceptación que durante la década de 1940 tuvo el cine mexicano en el resto de los países de la región, fenómeno que ha sido estudiado de manera sistemática por los historiadores del cine, especialmente en lo relativo a la llamada época de oro (1941 a 1945). Véase, por ejemplo, la Historia del cine mexicano de Emilio García Riera (1985) o la Historia del cine mexicano de David Viñas (1987).
} 
otras redes de acceso en internet o lo que se puede conseguir en los circuitos de reproducción ilegal, sin los cuales la investigación profesional del cine sería imposible en nuestros países.

San Andresito en Bogotá, Tepito en la Ciudad de México y otras zonas de venta de materiales cinematográficos especializados de origen ilegal son, al parecer, los espacios naturales donde la cinefilia especializada puede abrevar para acceder a lo que los circuitos comerciales de exhibición, la programación televisiva y los archivos fílmicos no llegan a cubrir. ${ }^{3}$

Esta situación tiene su correlato en la escasez de materiales bibliográficos de la región en las bibliotecas especializadas en cine, que se encuentran ya sea en los archivos fílmicos o en las escuelas de cine de cada país. El insularismo también significa que no contamos con ninguna biblioteca latinoamericana de cine con las proporciones de las que encontramos en las filmotecas de Amsterdam, Barcelona, Madrid, París, Turín o Valencia, no digamos Berkeley, Londres o Berlín, en las cuales hay suscripción a más de 100 revistas especializadas en teoría, historia y análisis cinematográfico (y no sólo a las revistas dedicadas a la crítica de los estrenos mensuales).

Éstas son las condiciones editoriales, archivísticas, bibliotecológicas, y de distribución y exhibición que determinan el insularismo de nuestras pesquisas epistemológicas sobre el cine en general, y sobre el cine latinoamericano en particular.

\section{La insularidad externa}

La segunda vertiente del insularismo es de carácter externo. Consiste en el aislamiento casi absoluto de los estudios latinoamericanos de cine con respecto a la tradición de los estudios de cine producidos en la tradición anglosajona, es decir, en los Estados Unidos, Canadá e Inglaterra. Y a este insularismo le corresponde, simétricamente, un insularismo de los estudios que se producen en (un sector considerable de) las universidades norteamericanas sobre el cine producido en la región latinoamericana, donde se reduce esta producción regional al estudio del cine militante, el cine político o, en el mejor de los casos, el cine de vanguardia, a pesar de que el cine es un medio de comunicación masiva, y que estas formas de cine no tienen la más mínima distribución en las salas comerciales de cine, y constituyen un sector de la producción fílmica al que el crítico Paul Julian Smith, de

\footnotetext{
3 Existen algunas iniciativas aisladas que contrarrestan, así sea de manera mínima, la falta de distribución de las películas latinoamericanas. Entre éstas se encuentran la reciente producción en DVD de colecciones de cine mudo por parte de los archivos fílmicos de Argentina, Brasil y Colombia; la creación de la Cinemateca Virtual de Chile; la existencia de algunos sitios de acceso al cine internacional, como www.mubi.com y el trabajo de distribuidoras independientes, como la mexicana Zafra Video.
} 
la Universidad de Cambridge, ha llamado el Cine de Festival (por ejemplo, directores como Amat Escalante, Carlos Reygadas o Fernando Eimbcke, para hablar de los directores mexicanos contemporáneos). ${ }^{4}$

El aislamiento de los estudios latinoamericanos frente a los estudios en lengua inglesa se debe a dos factores simultáneos. En primer lugar, en todos los países de la región se comparte un radical rechazo casi instintivo a todo lo que proviene de los Estados Unidos. Esta actitud se explica por razones históricas, pero éstas son completamente ajenas a la calidad de la investigación especializada producida en las universidades norteamericanas. Este aislamiento es muy doloroso si observamos la riqueza que tiene la tradición anglosajona en los terrenos estratégicos de los libros de texto universitarios, los estudios de campo sobre el público de cine y las reflexiones sistemáticas sobre ética y estética, especialmente en los estudios de los géneros clásicos y de las relaciones entre el cine y la filosofía, el derecho y la cultura popular.

Y en segundo lugar, este aislamiento de los estudios latinoamericanos frente a la tradición anglosajona se debe a que los materiales producidos originalmente en otras lenguas han llegado a Latinoamérica a través de las traducciones producidas en España. Aquí es inevitable mencionar el lugar estratégico que ha tenido la editorial Paidós durante las décadas de 1980, 1990 y los primeros 5 años de la década de 2000. Y también es necesario señalar la existencia de lo que podríamos llamar, sin duda, la terrible Catástrofe Paidós. A partir del momento en el que esta editorial española decidió suspender la colección de estudios sobre cine, en la región iberoamericana sufrimos una orfandad editorial que no ha sido cubierta por ninguna otra editorial. Seguramente debido a la crisis económica, incluso la editorial Cátedra, de Madrid, ha aminorado notablemente el ritmo de publicaciones en este terreno.

Un caso suficiente para ejemplificar esta catástrofe se puede observar en la traducción del Diccionario teórico y crítico del cine, de Jacques Aumont y Michel Marie, que seguramente habría sido traducido y distribuido planetariamente por Paidós, pero que ahora ha sido traducido en Buenos Aires en la editorial La Marca.

\footnotetext{
4 Este fenómeno sigue vigente, como se puede observar en el número de 2010 de la revista de LASA (Latin American Studies Association), dedicado a los estudios sobre cine latinoamericano producidos en los Estados Unidos. Sin embargo, ésta es la tendencia dominante, pero afortunadamente no es exclusiva, como puede observarse en trabajos recientes, como el número de The Americas dedicado al cine mudo latinoamericano (coordinado por Ana López); el estudio de Jeffrey M. Pilcher sobre Cantinflas y la modernidad, y el de Jeffrey Himpele sobre el cine andino; el trabajo de Zuzana Pick sobre el cine y la revolución mexicana, y muy especialmente el volumen colectivo sobre cine latinoamericano coordinado por Deborah Shaw. Ver las referencias bibliográficas al final de este trabajo.
} 
Aunque este libro llega a las librerías de Bogotá, sin embargo no tiene ninguna distribución en México y otros países de la región.

Este último elemento ha tenido un efecto crucial en la tradición epistemológica de los estudios sobre cine en Latinoamérica, pues en España siempre ha habido una gran atención a lo que se produce en ruso, italiano, holandés y, sobre todo, en francés. Pero hay una casi total indiferencia por traducir lo que se produce en inglés. Por esta razón, en nuestras universidades hay cierta familiaridad con terrenos de los estudios cinematográficos particularmente desarrollados en la tradición continental, como la narratología, la semiótica y las relaciones entre el cine y la historia (es decir, sobre la articulación entre la historia social y su representación en el cine de ficción).

Esta misma insularidad de origen histórico y extracinematográfico ha determinado que en la investigación producida en nuestras universidades ( $y$ en los espacios de la crítica cinematográfica, como las revistas especializadas) se adopte una actitud de rechazo a la teoría del cine, considerándola como un terreno inútil o, en el mejor de los casos, como un mal necesario. Esta indiferencia ante la teoría parece desprenderse del empleo que se hace en el habla cotidiana del término teórico no con fines descriptivos, sino como un adjetivo que es sinónimo de inútil o irrelevante. Esta trivialización del término es comprensible en el habla cotidiana, pero es inexplicable cuando determina las políticas universitarias en las disciplinas humanísticas, en particular en los estudios sobre cine, literatura o filosofía.

Así, ya que no existen programas de doctorado en teoría cinematográfica en ningún país latinoamericano, no es casual que en los programas de Doctorado en Teoría Literaria o en Literatura Hispánica (por ejemplo, en México) se prohíba a los estudiantes presentar proyectos de teoría literaria, exigiéndoles, en cambio, utilizar teorías y metodologías producidas en otras lenguas (con excepción, por cierto, de la lengua inglesa, que sigue siendo la lengua proscrita en los estudios humanísticos en la región).

\section{Paréntesis epistemológico}

Aquí es necesario detenerse un momento para señalar las características de la tradición humanística anglosajona, especialmente en los estudios cinematográficos. Se trata de una tradición de carácter inductivista, fuertemente casuística y heredera de los métodos inferenciales de las ciencias naturales, con profundas raíces pragmáticas. En esta tradición, donde la filosofía siempre ha estado en diálogo con la pedagogía, la literatura y la cultura popular, resulta natural que exista una fuerte tradición de estudios sobre la dimensión política de toda expresión estética, y donde de manera sistemática se pregunta por la articulación entre teoría y práctica, filosofía e historia, ética y estética. Y donde la enseñanza universitaria 
(especialmente en los terrenos de la historia, la teoría y el análisis) es considerada como una profesión que requiere sus propias metodologías de investigación.

En contraparte, la tradición continental tiende a estar ligada al desarrollo de la historiografía; es de carácter deductivista, fuertemente nomotética y heredera de los métodos de las ciencias sociales, con profundas raíces de naturaleza racionalista. En esta tradición, donde la filosofía ha estado alejada de los circuitos del poder político, se originó la polémica del realismo cinematográfico con el formalismo europeo. Y por el peso que en esta tradición tienen las ciencias sociales, incluso la misma semiología fue originalmente concebida como una rama de la psicología social. Ésta es la tradición que ha sido la dominante en la región iberoamericana, especialmente en su versión francesa.

Es por esta influencia proveniente de las ciencias sociales ---y muy especialmente de la historiografía europea--- que los trabajos más originales y de alcance universal que se han producido en lengua española están ligados al estudio político del cine de ficción (en Brasil), a la militancia política (en Argentina, Bolivia y Cuba), al cine documental (como en el trabajo de Pablo Corro y su equipo, en Chile) y a la historia del arte (como en los recientes trabajos de Ángel Faretta en Argentina).

También esta impronta de la tradición continental explica la presencia abrumadoramente mayoritaria de los historiadores en el campo de los estudios sobre cine en la región, lo cual amerita dirigir una mirada más detenida.

\section{El imperio de los historiadores}

Al observar la producción de libros y artículos especializados, congresos de investigadores y tesis de posgrado producidos en la región latinoamericana durante los últimos 50 años es abrumadora la presencia dominante de los historiadores. Tan sólo en el caso mexicano, en el período comprendido entre 1980 y 2005, de los poco más de 400 libros publicados sobre cine, más de 250 tratan sobre temas historiográficos, o bien fueron escritos por historiadores profesionales. ${ }^{5}$

Por supuesto, esto permite hablar de la existencia de una tradición en las aproximaciones que hacen los historiadores y, en general, los científicos sociales, al cine en la región. Los primeros congresos académicos de especialistas en cine (que datan de 1989) fueron convocado por el historiador Eduardo de la Vega, quien a su vez heredó el lugar que antes fue ocupado en la Universidad de Guadalajara por el periodista de origen español Emilio García Riera.

5 Este sorprendente fenómeno está documentado en el trabajo de Ángel Miquel y Lauro Zavala: "El placer de leer cine: 25 años de libros sobre cine en México (1980 - 2005)", en El placer de ver cine. Número especial de Tierra Adentro (Consejo Nacional para la Cultura y las Artes), núm. 141, agosto - septiembre 2006, 96-109. 
Esta tradición tiene una amplia gama de variantes. En la historiografía del cine latinoamericano encontramos, por supuesto, las aproximaciones a la historia nacional y regional a través del cine (Aleksandra Jablonska), la historia de los escritores que se interesaron por hacer crítica de cine o literatura con motivos cinematográficos (Ángel Miquel), la historia de la industria cinematográfica (Emilio García Riera), así como las historias regionales del cine (es decir, las microhistorias del cine, en la línea de José Luis González), las biografías de actores y las monografías de directores del cine nacional (Eduardo de la Vega) o la historia de la presencia de Eisenstein en México y su influencia en el cine de los años 40 y 50 (Aurelio de los Reyes), la historia y el rescate de las películas del cine mudo (en las filmotecas universitarias de Argentina, México y Venezuela), así como la historia de las adaptaciones de la literatura nacional en el cine internacional (en especial en el caso de Colombia), la historia del cine político e historiográfico sobre periodos específicos de la historia nacional (Clara Kriger sobre el cine peronista en Argentina) y la historia de la ciudad y del campo en el cine nacional (Rafael Aviña en México).

Esta diversidad de aproximaciones es extensiva a los estudios provenientes de las ciencias sociales. Por ejemplo, encontramos las aproximaciones antropológicas a los hábitos de consumo audiovisual (Néstor García Canclini en México) o sobre el público regional (Patricia Torres en Guadalajara y Norma Iglesias en Tijuana), así como las aproximaciones sociológicas al melodrama familiarista latinoamericano (Silvia Oroz en Argentina), la presencia de las mujeres delante y detrás de las cámaras (Julia Tuñón, Maricruz Castro y otras, en México) y una reflexión sobre la identidad regional en el cine latinoamericano (Paulo Antonio Paranaguá, desde París).

La creación de esta tradición académica en las ciencias sociales tiene su correlato en la creciente importancia que ha adquirido en los últimos diez años la producción, el estudio y la exhibición de un cine documental que ya no está limitado a las consignas del cine militante, y que explora terrenos tan atractivos como la presencia de las mujeres en el deporte, los testimonios de los ladrones de los años 50 y 60 o los campeones mundiales de boxeo de los años 60 y 70, así como los de quienes no emigran a los Estados Unidos. Estas nuevas formas del documental exigen nuevas herramientas de análisis, en consonancia con la lógica del cine transnacional. ${ }^{6}$

6 Una visión panorámica sobre este tema se encuentra en mi trabajo "El nuevo documental mexicano y las fronteras de la representación", que forma parte del libro recién publicado por la Universidad de Guadalajara, bajo la coordinación de Juan Carlos Vargas (2011). 


\section{Hacia el futuro}

La importancia que tiene la tradición contextual en los estudios cinematográficos en Latinoamérica es tan abrumadora (frente lo que podríamos llamar la incipiente tradición textual) que incluso los pocos autores norteamericanos que están traducidos al español son precisamente quienes han reseñado la historia de la teoría europea del cine, como Dudley Andrew en los años 70 y Robert Stam en el año 2000.

En contraste, quedan todavía sin ser traducidos trabajos tan relevantes en la tradición interpretativa anglosajona, en donde se integran las herramientas del análisis estructural o semiótico a proyectos de investigación sociohistórica. Éste es el caso, por ejemplo, de Sixguns and Society, el histórico trabajo de Will Wright sobre la estructura mitológica del western, donde se muestra el cambio ideológico que se produce al haber una fractura histórica (como la muerte de Kennedy en 1963), y que marca el inicio de lo que ahora conocemos como los Estudios Culturales, precisamente en un terreno medular de los Estudios Cinematográficos.

Tampoco está traducido ninguno de los trabajos de Stephen Prince sobre la amplitud estilística en la representación de la violencia en el cine, que permite distinguir la violencia clásica de la ultraviolencia hiperbólica y la hiperviolencia irónica, banalizada o distanciada al estilo brechtiano.

Sin duda, también sería deseable la traducción de materiales como The Moral of the Story (algo así como La moraleja del cuento), de Nina Rosenstadt, sobre la historia de la ética a través del cine y la literatura, y que ha tenido numerosos seguidores; Introduction to Documentary, de Bill Nichols, que es piedra angular de las discusiones contemporáneas sobre cine documental; ${ }^{7}$ Cognitive Semiotics de Warren Buckland, donde se integran las tradiciones de la semiótica y el cognitivismo. O el espléndido trabajo del mismo Buckland y Thomas Elsaesser sobre los métodos de análisis tradicionales y modernos, aplicados ejemplarmente en su Studying Contemporary American Films. A Guide to Movie Analysis.

Por último, no deja de sorprender que no hayan sido traducidos al español los trabajos de Noël Carroll o el volumen colectivo Post-Theory, del mismo Carroll y David Bordwell (que fue en 1996 el acta de defunción de la teoría lacanianoalthusseriana-marxista, y el nacimiento de la Teoría de Nivel Medio). Ni siquiera está traducido el análisis neoformalista de Iván el Terrible, de Kristin Thomspon o la poética del cine en Ozu, de David Bordwell. Y ninguna de las antologías de teoría y

\footnotetext{
7 Este hecho es aún más enigmático cuando la misma editorial Paidós tradujo uno de los primeros libros de Bill Nichols, Representing Reality, pero dejó sin traducir los dos volúmenes de teoría cinematográfica editados por el mismo Nichols, Movies and Methods I (1976) y Movies and Mehods II (1984).
} 
análisis del cine producidas después de la que publicó Bill Nichols en 1979, como las de Braudy y Cohen; Gledhill y Williams; Miller y Stam; Hill y Gibson o la más reciente, de Geiger y Rutsky.

Es igualmente sorprendente el hecho de que hasta la fecha sólo existe un par de libros de texto traducidos al español para estudiar el lenguaje cinematográfico. La admirable tradición pedagógica en la industria editorial norteamericana ha producido al menos dos docenas de libros de texto acompañados por fotografías en color en cada página y numerosos recuadros didácticos en cada capítulo, un DVD en cada ejemplar, una guía para el profesor, y ejercicios disponibles en un sitio de internet. Cuando estos materiales han sido traducidos al español se reducen a una versión muy sobria, sin ilustraciones ( $U n$ arte compartido (1987), de James Scott) o con fotogramas en blanco y negro (El arte cinematográfico, de David Bordwell y Kristin Thompson). Este concepto editorial empezó a cuajar en Francia tímidamente (es decir, con fotogramas de formato muy pequeño) con la publicación del libro de Laurent Jullier y Michel Marie, Lire les images de cinéma (2009).

Mientras tanto, siguen sin traducción al español los espléndidos libros de texto de análisis cinematográfico de Abrams, Barsam, Bobker, Boggs, Bone, Buckland, Corrigan y un larguísimo y apasionante etcétera que cubre todo el abecedario, y que se actualizan cada dos o tres años, incluyendo sus correspondientes guías para el profesor, su página en internet con materiales de apoyo didáctico, y su disco con secuencias selecccionadas para ser proyectadas durante el curso.

Esta indiferencia hacia las aproximaciones humanísticas, interdisciplinarias, didácticas y pragmáticas, tan características de la tradición anglosajona, han tenido como consecuencia que en los estudios sobre la historia del cine en Iberoamérica ha dominado la aproximación textual (Roman Gubern o Emilio García Riera) por encima de la aproximación intertextual a la historia del cine (como la influencia de la tecnología en el desarrollo del lenguaje audiovisual, al estilo de Barry Salt).

$\mathrm{Y}$ es evidente también que en los estudios sobre cine y literatura sigue dominando en Iberoamérica la teoría de la adaptación (y con ella el problema de la fidelidad al texto literario) por encima del concepto de traducción intersemiótica (y el reconocimiento de la autonomía estética del cine frente a la literatura).

En síntesis, parece seguir dominando el uso del cine con fines disciplinarios o instrumentales por sobre los fines interpretativos y analíticos desde una perspectiva estética, interdisciplinaria y transnacional (con excepción de lo que ocurre en Brasil y en España). En otras palabras, el estudio del cine en la región sigue estando dominado por el interés que tiene como industria cultural (en las carreras de 
comunicación) más que como una forma de arte (en el campo de las humanidades).

Hasta la fecha, el uso del cine con fines disciplinarios sigue estando por encima de los procesos de interpretación. Es por esto que todavía no existe un Doctorado en Estudios Cinematográficos en ninguna universidad latinoamericana.

Y sin embargo, conviene señalar el surgimiento de una tradición de reflexión teórica y de análisis en nuestra lengua, producida precisamente en algunos grupos de investigadores españoles. Mencionemos, por ejemplo, los encuentros internacionales convocados por la Asociación Cultural Trama y Fondo, presidida por Jesús González Requena (en Valladolid), que desde el año 1995 publica una revista semestral de investigación interdisciplinaria. También es notable la organización del congreso internacional dedicado al Análisis Cinematográfico, convocado por un importante grupo de investigadores españoles, entre los que es necesario mencionar a Francisco Javier Gómez Tarín (en Valencia) y Javier Marzal Felici (en Madrid), quien además coordina, desde el año 2000, una conocida serie bibliográfica de análisis de películas particulares (publicada en Valencia).

Esta tradición humanística gana cada día más fuerza, y no sólo establece un contrapeso a la tradición continental, sino que ha logrado equilibrar ambas tradiciones (continental y anglosajona). De esa manera, esta nueva tradición española, en conjunción con la tradición italiana liderada por Francesco Casetti (quien siempre ha recomendado la posibilidad de adecuar los recursos de diversas tradiciones metodológicas a las necesidades de cada proyecto), lleva a la creación de lo que se podría considerar como la escuela mediterránea en la teoría y el análisis cinematográfico.

Algo similar empieza a ocurrir en algunos espacios de la investigación en las universidades latinoamericanas. Menciono el caso del grupo de investigación surgido en la Universidad de Guadalajara en 2009, bajo la coordinación de Patricia Torres y Álvaro Fernández, que tiene un carácter interdisciplinario y donde hay interés por campos muy diversos de la investigación cinematográfica, lo mismo por los estudios antropológicos sobre el público de cine que por el análisis estructural del suspenso narrativo o las formas que adoptan los géneros clásicos en el cine latinoamericano. Hasta la fecha este grupo ha convocado a tres congresos internacionales, estableciendo vínculos entre los investigadores de cine latinoamericano en México, Francia y España.

\section{Conclusión}

En América Latina todo está por hacerse. Ya bien entrado el siglo XXI todavía no contamos con una revista que compita con Dirigido Por y Versión Original en España, y la veintena de revistas especializadas de alcance transnacional que publica 
la editorial Intellect en Inglaterra (Journal of Chinese Movies o el Journal of French Cinema, etc.). O con colecciones de libros como las publicadas desde hace varias décadas por el British Film Institute (BFI) o en la editorial Routledge, todos ellos en Inglaterra.

La organización de encuentros de investigadores latinoamericanos y la creación de asociaciones de investigadores de la región es un buen signo de cambio. En el primer campo mencionemos el convocado por el Instituto de Investigaciones Estéticas de la Universidad Nacional de Colombia (con apoyo de la Dirección de Cinematografía del Ministerio de Cultura), en octubre de 2010, y el convocado por la Red INAV (Red de Investigadores de Narrativas Audiovisuales), también con sede en Bogotá, y realizado en 2010 en la Ciudad de México.

Y en cuanto a las asociaciones de investigadores, en 1995 se creó SOCINE (Sociedade Brasileira de Estudos de Cinema e Audiovisual); en 2008 se creó SEPANCINE (Seminario Permanente de Análisis Cinematográfico / Asociación Mexicana de Teoría y Análisis Cinematográfico) y en 2009 se creó ASAECA (Asociación Argentina de Estudios sobre Cine y Audiovisual), cada una de las cuales realizan regularmente congresos de investigadores en los que participan especialistas de los demás países de la región.

Todo ello puede contribuir a crear un clima de discusión que lleve a formular balances de lo que se ha hecho hasta ahora y lo que se podría llegar a hacer en la región (como la escritura de una Historia del Cine Mundial, desde una perspectiva hispanoamericana). El futuro está a la vuelta de la esquina. Pero es necesario empezar a construirlo cuanto antes.

\section{Referencias bibliográficas}

Abrams, Nathan; Ian Bell; Jan Udris: Studying Film. London, Arnold, 2001

Andrew, Dudley: Las principales teorías cinematográficas. Traducción de Homero

Alsina Thevenet. Revisión de Joaquim Romaguera i Ramió. Barcelona, Gustavo Gili, 1978 (1976)

: Concepts in Film Theory. Oxford University Press, 1984

Aumont, Jacques y Michel Marie: Diccionario teórico y crítico del cine. Buenos Aires, La Marca, 2006 (2001)

Aviña, Rafael: Tierra brava. El campo visto por el cine mexicano. Edición iconográfica de Susana Casarín. México, Instituto Mexicano de Cinematografía / Compañía Nacional de Subsistencias Populares, 1999.

Barsam, Richard: Looking at Movies. An Introduction to Film. New York, W.W. Norton, 2004 
Bobker, Lee: Elements of Film. New York, Harcourt Brace and Jovanovich, 1977

Boggs, Joseph M.: The Art of Watching Films. Mountain View, California, Mayfield Publishing Company, 1991

Bone, Jan \& Ron Johnson: Understanding the Film. An Introduction to Film Appreciation. Lincolnwoods, Illinois, 1996

Bordwell, David: La narración en el cine de ficción. Barcelona, Paidós, 1996 (1985)

Bordwell, David \& Noël Carroll, eds.: Post-Theory. Reconstructing Film Studies. Madison, The University of Wisconsin Press, 1996

Bordwell, David \& Kristin Thompson: El arte cinematográfico. Una introducción. Barcelona, Paidós, 1995 (1993)

Braudy, Leo \& Marshall Cohen, eds.: Film Theory and Criticism. Introductory Readings. Oxford University Press, 1999

Buckland, Warren: The Cognitive Semiotics of Film. Cambridge, Cambridge University Press, 2000

Buckland, Warren y Thomas Elsaesser: Studying Contemporary American Films. A Guide to Movie Analysis. London, Arnold / New York, Oxford University Press, 2002

Carroll, Noël: Engaging the Moving Image. New Haven and London, Yale University Press, 2003

Casetti, Francesco: Teorías del cine, 1945 - 1990. Madrid, Cátedra, 1994 (1993)

Casetti, Francesco y Federico di Chio: Cómo analizar un film. Paidós, Barcelona, 1991 (1990)

Castro, Maricruz: “Género y estudios cinematográficos en México” en Ciencia Ergo Sum. Revista científica multidisciplinaria, Universidad Autónoma del Estado de México, Toluca, vol. 16, núm. 1, marzo-junio de 2009, 64-70

Corrigan, Timothy \& Patricia White: The Film Experience. An Introduction. Boston, Bedford / St. Martin's, 2009

Corro, Pablo; Carolina Larráin; Maite Alberdi; Camila van Diest: Teorías del cine documental chileno 1957 - 1973. Santiago, Instituto de Estética, Pontificia Universidad Católica de Chile, 2007

De la Vega Alfaro, Eduardo (coordinador): Microhistorias del cine en México. Universidad de Guadalajara / Cineteca Nacional / Dirección General de Actividades Cinematográficas de la UNAM / Instituto Mexicano de Cinematografía / Instituto Mora, 2001

De los Reyes, Aurelio: El nacimiento de ¡Que viva México! México, Instituto de Investigaciones Estéticas, Universidad Nacional Autónoma de México, 2009

Faretta, Ángel: El concepto del cine. Buenos Aires, Editorial Djaen, 2005 
Fernández Reyes, Álvaro: Santo, el enmascarado de Plata. Morelia, El Colegio de Michoacán / Consejo Nacional para la Cultura y las Artes, 2004

: Crimen y suspenso en el cine mexicano, 1946 - 1955. Morelia, El Colegio de Michoacán, 2007

García Canclini, Néstor (coordinador): Los nuevos espectadores. Cine, televisión y video en México. México, Instituto Mexicano de Cinematografía, 1994

García Riera, Emilio: Historia del cine mexicano. México, Secretaría de Educación Pública, 1985

García Saucedo, Jaime: Diccionario de literatura colombiana en el cine. Bogotá, Panamericana Editorial, 2003

Geiger, Jeffrey \& R. L. Rutsky, eds.: Film Analysis. A Norton Reader. New York, W. W. Norton, 2005

Gledhill, Christine \& Linda Williams, eds.: Reinventing Film Studies. London, Arnold, 2000

Gómez Tarín, Francisco Javier: Discursos de la ausencia. Elipsis y fuera de campo en el texto filmico. Valencia, Generalitat Valenciana / La Filmoteca, 2006

González Requena, Jesús: Clásico, manierista, postclásico: los modos del relato en el cine de Hollywood. Madrid, Castilla Ediciones, 2006

Hill, John \& Church Gibson (eds.): The Oxford Guide to Film Studies. Oxford University Press, 2000

Himpele, Jeffrey: Circuits of Culture: Media, Politics, and Indigenous Identity in the Andes. The University of Minnesota Press, 2007.

Jablonska, Aleksandra: Cristales del tiempo: pasado e identidad de las películas mexicanas contemporáneas. México, Universidad Pedagógica Nacional, 2009

Jullier, Laurent y Michel Marie: Lire les images de cinéma. Paris, Larousse, 2009

Kriger, Clara: Cine y peronismo. El estado en escena. Buenos Aires, Siglo XXI Editores, 2009

Machado, Arlindo: El sujeto en la pantalla. La aventura del espectador, del deseo a la acción. Barcelona, Gedisa, 2009

Marzal Felici, Javier: Guía para ver y analizar Citizen Kane. Valencia, Naus Llibres, 2000

---------: Cómo se lee una fotografía. Interpretaciones de la mirada. Madrid, Cátedra, 2007

Miller, Toby \& Robert Stam (eds.): A Companion to Film Theory. Oxford, Blackwell, 2004

Nichols, Bill (ed.): Movies and Methods, Vol. I. Berkeley, University of California, 1976

-------- (ed.): Movies and Methods, Vol. II. Berkeley, University of California, 1985 
Nichols, Bill: Ideology and the Image. Social Representation in the Cinema and Other Media. Bloomington, Indiana University Press, 1981

: La representación de la realidad. Cuestiones y conceptos sobre el documental. Barcelona, Paidós, 1997. Traducción de Representing Reality (1991)

--------: Introduction to Documentary. Bloomington, Indiana University Press, 2001. (Second edition, 2010).

Onaindia, Mario: El guión clásico de Hollywood. Barcelona, Paidós, 1996

Oroz, Silvia: El cine de lágrimas de América Latina. México, UNAM, 1995

Paranaguá, Paulo Antonio: Tradición y modernidad en el cine de América Latina. Madrid, Fondo de Cultura Económica, 1996

Pick, Zuzana M.: Constructing the Image of the Mexican Revolution: Cinema and the Archive. The University of Texas Press, 2010.

Pilcher, Jeffrey M.: Cantinflas and the Chaos of Mexican Modernity. Washington, Scholarly Resources, 2001

Prince, Stephen: Classical Film Violence. Designing and Regulating Brutality in Hollywood Cinema 1930 - 1968. New Brunswick, Rutgers University Press, 2003

Rosenstadt, Nina: The Moral of the Story. An Introduction to Questions of Ethics and Human Nature. Mountain View, California, Mayfield Publishing Company, 1994

Russo, Eduardo A.: Diccionario de cine. Buenos Aires, Paidós, 1998

Salt, Barry: Film Style and Technology: History and Analysis. $2^{\text {nd }}$ ed., London, Starwood, 1992

Scott, James F.: El cine, un arte compartido. Pamplona, Ediciones de la Universidad de Navarra, 1979 (1975)

Shaw, Deborah (ed.): Contemporary Latin American Cinema. Breaking the Global Market. Rowman \& Littlefield Publishers, 2007

Smith, Paul Julian: "Revisiones del cine mexicano" en Casa del Tiempo, revista de la Universidad Autónoma Metropolitana, núm. 29, marzo de 2010, 46-49. Este texto está accesible en línea, en el sitio de Casa del Tiempo.

Stam, Robert: Teoría del cine. Una introducción. Barcelona, Paidós, 2002 (2000).

Stam, Robert; Robert Burgoyne, Sandy Flitterman-Lewis: Nuevos conceptos de la teoría del cine. Barcelona, Paidós, 1999 (1993)

Thompson, Kristin: Eisenstein's Ivan the Terrible. A Neoformalist Analysis. Princeton University Press, 1981

: Breaking the Glass Armor. Neoformalist Film Analysis. Princeton University Press, 1988 
Tuñón, Julia: Mujeres de luz y sombra en el cine mexicano. La construcción de una imagen, 1939 - 1952. México, El Colegio de México / Instituto Mexicano de Cinematografía, 1998

Vargas, Juan Carlos (coordinador): Tendencias del cine iberoamericano en el nuevo milenio. Argentina, Brasil, España y México. Guadalajara, Universidad de Guadalajara, 2011, $536 \mathrm{p}$.

Viñas, David: Historia del cine mexicano. México, Universidad Nacional Autónoma de México, 1987

Wolf, Sergio: Cine / Literatura. Ritos de pasaje. Buenos Aires, Paidós, 2001

Wright, Will: Sixguns and Society. A Structural Study of the Western. Berkeley, University of California, 1975

Xavier, Ismail: El discurso cinematográfico. La opacidad y la transparencia. Buenos Aires, Manantial, 2005 (1977) 